\title{
Analyzing the Competitiveness of the Greek Sea Bream Exports in the European Union Market
}

\section{Oikonomou $\mathrm{A}^{*}$ and Polymeros $\mathrm{K}$}

Department of Ichthyology and Aquatic Environment, University of Thessaly, Fytokou Street, Nea lonia, Greece

\begin{abstract}
This study investigates the evolution of the competitiveness level of the Greek sea bream exports in the EU-27, by implementing the Revealed Export Competitive advantage index. In particular, the major importing and exporting countries of gilthead sea bream were identified. Results reveal that the main importing markets of the Greek sea bream are Italy, France, Portugal, Spain, United Kingdom and Germany. Also, an important emerging market is Romania. While, competing countries are Spain, Turkey, France, Italy, Malta and Croatia. However, the level of competition, among the exporting countries, is continuously changing, indicating a high competitive market environment. Thus, policy and marketing strategies should be cautiously designed, in order to fulfill the rapid changes at this demanded market.
\end{abstract}

Keywords: Sea bream; Exports; Competitiveness; Greece; European union; Revealed export competitive advantage index

\section{Introduction}

Aquaculture is considered to be one of the fastest growing sectors of production of animal products. According to the International Food and Agriculture Organization (FAO), aquaculture worldwide amounted to 62.7 million tons, rising by $6.2 \%$ from 59 million tons in 2011. It is estimated that the top 20 fish production countries produced $95 \%$ of farmed fish worldwide. The production of aquaculture products is estimated at about 66.5 million tons in 2011. The contribution of aquaculture to global fisheries production has increased from $3.22 \%$ in 1950 to more than $40 \%$ while aquaculture production increased rapidly at the late 80's from 11 million tons in 1985 to 62.7 million tons in 2011. On the contrary, production of collective fishing stabilized in recent years (range at 90 million tons) with an increase up to 93 million tons in 2011. The marine fish farming is an important sector of economic activity of the Greek fishing production, as well in terms of size as in terms of comparative advantages exhibited by Greece [1].

Although Greece is relatively small $\left(131,940 \mathrm{Km}^{2}\right)$, it is blessed with an extensive coastline of $15,000 \mathrm{Km}$ with favorable climatic characteristics that could support the growth of marine aquaculture, which has evolved into one of the most developing sectors over the last decade.

The ever growing significance of aquaculture in Greece, is attributed to the following factors: a) the reduction of species of collecting fishing due to pollution-water contamination, overfishing and rising fuel costs, b) the impressive development of farming technology in the various species of aquatic organisms, after the 2nd World War, c) the key solution is aquaculture to meet the growing demand in recent years catches.

Gilthead sea bream (Sparus aurata) and sea bass (Dicentrarchus labrax), the two main finfish species that make up this sector today have only been farmed since the beginning of the 1980s. These two species make up over 95 percent of the total production in Greece, in order of importance in terms of tonnage produced.

According to the above, Greece undoubtedly plays a dominant role in the production of aquaculture products in Europe and particularly in the production of sea bream, since its production ranges over the half (54\%) among the other European countries. Thus, the sector of aquaculture as one of the most important sectors of the food industry seems to be subjected to intense competitive pressures and therefore is a very interesting project for economic analysis.

Greece ranks first in production among European Union and Mediterranean countries of commercial aquaculture finfish species and the sector ranks second in exports of "food- beverages", contributing significantly to the country's national economy [2].

In recent years competitiveness has been widely used from different perspectives as a term in economic research and economic policy, although its definition has not been widely agreed yet. Competition involves a number of traders in a particular market, as well as the market share, the sustainability of that share and profitability. According to Porter [2] the level of competition of a sector in a particular market depends on the following five key factors: a) the number of new entrants, b) the existence of substitute products, c) the bargaining power of suppliers, $d$ ) the bargaining power of buyers and e) Competition between existing firms in the market. Competition is dynamic and constantly evolving, as new products, new methods of marketing, new production processes and new markets appear [3]. According to Kim and Marion [4], the degree of competitiveness in the domestic market is positively related to the ability of firms to compete in international markets, although strong domestic competitiveness is the most appropriate background to prepare companies to be competitive and viable in the globalized market.

The purpose of this study is to identify and to analyze the competitive position of exports of Greek gilthead sea bream (Sparus aurata) towards the EU market, since gilthead sea bream is one of the main exporting products of Greece in this market.

The investigation was carried out initially by: a) the identification of the major importing countries-markets, b) the determination of

${ }^{*}$ Corresponding author: Oikonomou A, Department of Ichthyology and Aquatic Environment, University of Thessaly, Fytokou Street, Nea Ionia, Greece, Tel: +302421093003; E-mail: aoikonom@uth.gr

Received April 21, 2015; Accepted May 11, 2015; Published May 18, 2015

Citation: Oikonomou A, Polymeros K (2015) Analyzing the Competitiveness of the Greek Sea Bream Exports in the European Union Market. J Glob Econ 3: 145. doi:10.4172/2375-4389.1000145

Copyright: (๑ 2015 Oikonomou A, et al. This is an open-access article distributed under the terms of the Creative Commons Attribution License, which permits unrestricted use, distribution, and reproduction in any medium, provided the original author and source are credited. 
the key competing countries - exporting, worldwide. Subsequently, it attempted the estimation of the selling prices of competing countries, to the major importing countries and finally it concluded by the evaluation of results for the evolution of the degree of competitiveness in each of the major importing markets.

\section{Methods and Materials}

According to the literature different indices have been used by many researchers to investigate the degree of competitiveness of national economies, industries, sectors and products. The following are the most important of them.

According to [5-9], the index of the Revealed Comparative Advantage (RCA) has been used to describe whether or not a country has a comparative advantage in a particular industry sector or product through trade, without analyzing the main sources of this comparative advantage. Defined as the ratio of the share of exports of a product or an industry sector of a country in the global market, to the share of total exports of all products or industry sectors of the country in the same market, RCA index imprinted as follows:

$$
R C A i j=(x i j / X j) /(x i w / X w)
$$

Where, $R C A i j$ : revealed comparative advantage index for product or sector $i$ of country $j$

$x i j$ : the exports of the product or sector i of country

$X j$ : total exports of country j

Xiw: the exports of the product or sector i worldwide

$X w$ : total exports worldwide

Whether or not a country has comparative advantage within a product category depends on the value of the index. Therefore, when value of the Revealed Competitive Advantage index is greater than 1, then the under the study product has a comparative advantage, whereas for lower values it has a comparative disadvantage $[7,10]$.

Modification of the above index is the Revealed Export Competitive Advantage index (RXCA), which calculates the competitive advantage of a country for a specific product, not on the global market, but within a specific target market and is expressed as follows:

$$
R X C_{i j}=A_{j}\left(X_{i j} / \sum_{i} X_{i j}\right) /\left(\sum_{j} X_{i j} / \sum_{i} \sum_{j} X_{i j}\right)
$$

The two indices RXA and RMA can take values from 0 to infinity, while values of RTA is either

$X$ : the export values

$i$ : the countries under study

$j:$ the products under study

This approach enables us to investigate the competitive position of a country's exports to specific markets, making it possible both to identify the main import markets, and secondly the degree of competitiveness within these markets. Moreover, by this modification it becomes a feasible process to identify major export competitor countries in these import markets.

When the value of RXCA index is greater than 1, then the country under study presents a competitive advantage, contrary to the values lower than 1 showing a competitive disadvantage.
Another way of investigating the degree of competitiveness according to Havrila and Gunawardan [6] is to utilize the indices of comparative advantage of Vollrath, which are the following:

i) The Relative Export Advantage Index (RXA)

ii) The Relative Import Advantage Index (RMA)

iii) The Relative Advantage Trade Index (RTA)

iv) The Relative Competitiveness Index (RC)

The Vollrath's indices imprinted as follows:

$$
R X A i j=(X i j / X n j) /(X i r / X n r)
$$

Positive or negative. Therefore, when the values of RXA and RMA indices are greater than 1 we have relevant export or import advantage respectively, while when they are lower than 1 we have export or import defect.

As for the values of RTA index when they are positive we have relevant commercial advantage, while when they are negative we have commercial disadvantage respectively $[7,11]$

The importance of Vollrath's indices is that they help us to distinguish a product/country between other products/countries, thus avoiding double counting in global trade [7].

This study was conducted by picking out the Relevant Export Competitive Advantage Index (RXCA) from all the above list of indices applied for the major importing countries markets of the EU-27.

Utilizing the mathematical operation Values to Quantities, the Gilthead Sea Bream's sale prices of competitive countries within the major importing countries of the EU-27, were compared.

$$
\begin{aligned}
& P i j=V i j / Q i j \\
& R M A i j=(M i j / M n j) /(M i r / M n r) R T A i j=R X A i j-R M A i j \\
& R C i j=\operatorname{Ln}(R X A i j)-\operatorname{Ln}(R M A i j)
\end{aligned}
$$

$X$ stands for the exports $M$ stands for the imports $i$ stands for the product

$j$ stands for the country

$n$ is the rest of the products

$r$ the rest countries

$P$ : the prices

$V$ : values

$Q$ : quantities $i$ : the country $j$ : the product

In this research, the values and quantities of the Greek fisheries and aquaculture products' exports and imports, particularly Gilthead Sea Bream, are the same as for the other countries of the EU-27 market, determined since 2000 up to 2013, derived from the Eurostat online database services at http://epp.eurostat.ec.europa.eu/newxtweb/

Categories and subcategories ranking products with 4-digit and 8-digits encoding were searched. Specifically, the codes used were:

0302: Fish, fresh or chilled, excluding fish fillets and other fish meat (category of fresh fish or chilled).

03026995 and 03028530 (since 2012): Fresh or chilled gilthead sea bream "Sparus aurata" (category fresh or chilled gilthead sea bream). 


\section{Results}

In 2008, the exports value of Greek sea bream reached approximately 138.25 million euros. Also, it has been found that within the seven major subcategories of Greek fishery and aquaculture products, subcategory "0302" (fish, fresh or chilled) occupies the largest share of Greek exports to the EU-27 market thus making Greece one of the main countries involved in this market. Specifically, the subcategory "0302", by 250 million euros in 2000 reached 409 million EUR in 2012, which means an increase of $39 \%$. The value of exports of Greek sea bream in the EU-27 market follows a continuous upward trend between 2005 and 2011 , with a reduction of $11 \%$.

In 2012, by 204 million euros in 2011, fell to 184 million euros. While the volume of Greek exports in sea bream has increased since 2000, from 19.000 tons to 43.000 tons in 2012, which means an increase of $55 \%$.

This research reveals that: the main importing countries-markets among the EU-27 are Italy, France, Portugal, Spain, the United Kingdom and Germany, with Romania as an emerging market. These countries are basically the main destinations of Greek exports. In particular, the Italian sea bream market occupies the largest share by an increasing turnover of 67 million euros in 2009 to 95 million euros in 2013, followed by France and Portugal, United Kingdom and Germany which maintain their rates on an upward trend. Spain has reduced its imports in sea bream the last two years. Romania is a new interesting market.

Moreover, the competitive countries of Greece in the EU-27 market among all countries worldwide for the last 6 years are Spain, Turkey, France, Italy, Malta and Croatia. Especially the Greek sea bream occupies the largest share by a turnover of 120 million euros in 2009 to 160 million euros in 2013, followed by Spain and Turkey. In particular, Turkey has increased its exports of sea bream in EU-27, reaching 20.6 million euros in 2013. France and Italy firmly maintain their rates while Malta and Croatia are new competitor countries in sea bream. The supremacy of Greece is clearly seen against the rest, for the period between 2008 and 2013.

Subsequently, we will investigate the competitiveness of Greek sea bream in the markets of Italy, France, Portugal, the United Kingdom, Germany, Spain and Romania compared to sea bream of Italy, France, Spain, Turkey, the Netherlands, Malta and Croatia by using RXCA index. This index for each import market is translated as a fraction with numerator the imports of that market in sea bream from another country by imports of "0302" in the same country and denominator imports of that market of sea bream in EU-27 by imports of " 0302 " in EU-27.

E.g. For the import market of Italy and Greece as the export country, the RXCA index formed as below:

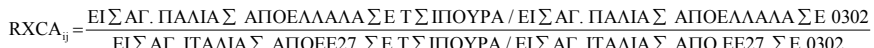

The Annex at the end of this article contains the tables comprising of the detailed data about the competitiveness indices, also the graphs which depict its fluctuation, as well as the tables of the selling prices (euros $/ \mathrm{kg}$ ) of sea bream in the seven import markets of EU-27, for two periods: 2000- 2007 and 2008-2013. The period 2008-2013 which is of the most interest is analyzed below.
The Italian market is the largest market for the Greek sea bream, which holds from 2000 to 2013 the largest market share. Specifically on average between 2008 and 2013, Greece has an RXCA index of 3 with a decrease of $15 \%$; Turkey has a value of 2.81 and an increase of $38 \%$, while Malta has the highest competitive advantage around 5 but a decrease of 29\% (Table 1 and Figure 1).

\begin{tabular}{|c|c|c|c|c|c|c|c|}
\hline Year & Greece & Spain & France & Turkey & Netherlands & Croatia & Malta \\
\hline 2000 & 2,88 & 0,28 & 0,18 & 0,46 & 0,01 & 0,20 & 7,40 \\
\hline 2001 & 3,37 & 0,35 & 0,19 & 0,31 & 0,01 & 0,32 & 6,88 \\
\hline 2002 & 3,38 & 0,41 & 0,17 & 0,49 & 0,01 & 0,48 & 8,38 \\
\hline 2003 & 3,41 & 0,40 & 0,15 & 0,83 & - & 0,51 & 7,16 \\
\hline 2004 & 3,26 & 0,28 & 0,19 & 0,85 & - & 0,53 & 5,91 \\
\hline 2005 & 3,48 & 0,29 & 0,19 & 1,48 & 0,01 & 1,00 & 4,53 \\
\hline 2006 & 3,78 & 0,26 & 0,23 & 1,09 & 0,11 & 1,15 & 6,45 \\
\hline 2007 & 3,17 & 0,30 & 0,16 & 1,83 & 0,06 & 0,79 & 6,01 \\
\hline 2008 & 3,42 & 0,33 & 0,22 & 2,14 & 0,08 & 0,73 & 5,98 \\
\hline 2009 & 3,18 & 0,38 & 0,26 & 2,31 & 0,04 & 1,07 & 5,65 \\
\hline 2010 & 2,92 & 0,29 & 0,22 & 3,47 & 0,15 & 1,16 & 5,07 \\
\hline 2011 & 2,67 & 0,29 & 0,25 & 3,04 & 0,22 & 0,84 & 4,72 \\
\hline 2012 & 2,60 & 0,33 & 0,20 & 2,77 & 0,19 & 1,11 & 4,25 \\
\hline 2013 & 2,90 & 0,28 & 0,23 & 2,95 & 0,13 & 1,13 & 4,26 \\
\hline Max 2000-2007 & 3,78 & 0,41 & 0,23 & 1,83 & 0,11 & 1,15 & 8,38 \\
\hline Min 2000-2007 & 2,88 & 0,26 & 0,15 & 0,31 & 0,01 & 0,20 & 4,53 \\
\hline $\begin{array}{l}\text { Average 2000- } \\
2007\end{array}$ & 3,33 & 0,33 & 0,19 & 1,07 & 0,06 & 0,67 & 6,46 \\
\hline $\begin{array}{l}\text { Variation } \% \\
2000-2007\end{array}$ & 10,19 & 6,58 & $-8,87$ & 298,43 & 923,29 & 299,55 & $-18,79$ \\
\hline $\begin{array}{l}\text { Std. Deviation } \\
2000-2007\end{array}$ & 0,26 & 0,06 & 0,02 & 0,53 & 0,04 & 0,33 & 1,15 \\
\hline Max 2008-2013 & 3,42 & 0,38 & 0,26 & 3,47 & 0,22 & 1,16 & 5,98 \\
\hline Min 2008-2013 & 2,60 & 0,28 & 0,20 & 2,14 & 0,04 & 0,73 & 4,25 \\
\hline $\begin{array}{l}\text { Average 2008- } \\
2013\end{array}$ & 3,01 & 0,33 & 0,23 & 2,81 & 0,13 & 0,94 & 5,11 \\
\hline $\begin{array}{l}\text { Variation \% } \\
2008-2013\end{array}$ & $-15,15$ & $-15,47$ & 3,50 & 37,77 & 72,74 & 54,84 & $-28,72$ \\
\hline $\begin{array}{l}\text { Std. Deviation } \\
2008-2013\end{array}$ & 0,31 & 0,04 & 0,02 & 0,49 & 0,07 & 0,18 & 0,72 \\
\hline
\end{tabular}

Table 1: RXCA index-Market of Italy.

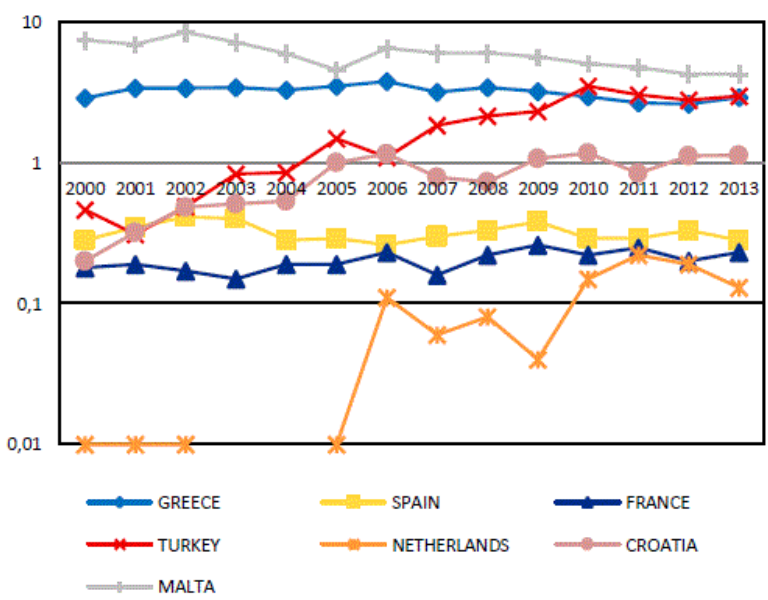

Figure 1: RXCA index-Market of Italy. 
Citation: Oikonomou A, Polymeros K (2015) Analyzing the Competitiveness of the Greek Sea Bream Exports in the European Union Market. J Glob Econ 3: 145. doi:10.4172/2375-4389.1000145

Page 4 of 10

For the market of France, it is found that the Greek sea bream holds the first place of import value share since 2000, followed by Spanish sea bream. Greece has a very high rate of $12.5 \%$ over the six years from 2008 to 2013 with a steady decrease in competitiveness index 7\%. Spain has an index of 2.8, but an increase of $42 \%$. For The Turkish sea bream we cannot draw safe conclusions due to minimum required trading data (Table 2 and Figure 2).

\begin{tabular}{|c|c|c|c|c|c|c|c|}
\hline Year & Greece & Italy & Spain & Turkey & Netherlands & Croatia & Malta \\
\hline 2000 & 28,03 & 0,74 & 3,59 & - & 0,59 & - & - \\
\hline 2001 & 24,71 & 0,03 & 2,14 & - & 0,10 & - & - \\
\hline 2002 & 20,27 & 0,23 & 1,86 & 0,57 & 0,11 & - & - \\
\hline 2003 & 18,01 & 0,28 & 2,26 & - & 0,18 & - & - \\
\hline 2004 & 16,74 & 0,28 & 1,95 & - & 0,31 & - & - \\
\hline 2005 & 16,71 & 1,12 & 2,31 & - & 0,17 & - & - \\
\hline 2006 & 16,27 & 0,55 & 2,30 & - & 0,17 & - & - \\
\hline 2007 & 13,23 & 0,32 & 2,56 & - & 0,56 & - & - \\
\hline 2008 & 13,32 & 1,67 & 2,34 & 7,89 & 0,30 & - & - \\
\hline 2009 & 13,79 & 1,74 & 2,97 & - & 0,19 & - & - \\
\hline 2010 & 13,97 & 2,03 & 3,10 & 9,27 & 0,22 & - & - \\
\hline 2011 & 12,85 & 2,56 & 2,30 & - & 0,49 & - & - \\
\hline 2012 & 10,99 & 1,88 & 2,84 & 23,95 & 0,57 & - & - \\
\hline 2013 & 12,38 & 3,57 & 3,32 & 6,97 & 0,99 & - & - \\
\hline Max 2000-2007 & 28,03 & 1,12 & 3,59 & 0,57 & 0,59 & - & - \\
\hline Min 2000-2007 & 13,23 & 0,03 & 1,86 & 0,57 & 0,10 & - & - \\
\hline $\begin{array}{l}\text { Average 2000- } \\
2007\end{array}$ & 20,63 & 0,57 & 2,73 & 0,57 & 0,34 & - & - \\
\hline $\begin{array}{l}\text { Variation \% } \\
2000-2007\end{array}$ & $-52,79$ & $-56,99$ & $-28,66$ & - & $-4,86$ & - & - \\
\hline $\begin{array}{l}\text { Std. Deviation } \\
2000-2007\end{array}$ & 4,89 & 0,34 & 0,54 & - & 0,20 & & \\
\hline Max 2008-2013 & 13,97 & 3,57 & 3,32 & 23,95 & 0,99 & - & - \\
\hline Min 2008-2013 & 10,99 & 1,67 & 2,30 & 6,97 & 0,19 & - & - \\
\hline $\begin{array}{l}\text { Average 2008- } \\
2013\end{array}$ & 12,48 & 2,62 & 2,81 & 15,46 & 0,59 & - & - \\
\hline $\begin{array}{l}\text { Variation \% } \\
2008-2013\end{array}$ & $-7,08$ & 113,41 & 41,77 & $-11,68$ & 229,23 & - & - \\
\hline $\begin{array}{l}\text { Std. Deviation } \\
2008-2013\end{array}$ & 1,10 & 0,72 & 0,41 & 8,01 & 0,30 & - & - \\
\hline
\end{tabular}

Table 2: RXCA index-Market of France.

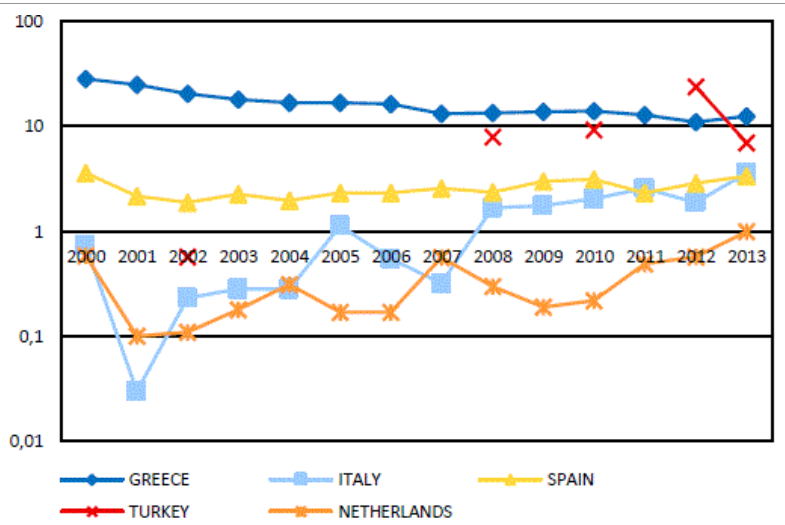

Figure 2: RXCA index-Market of France.
In the market of Portugal, the Greek sea bream which holds the second place of import value share has a RXCA index of average 3 with a rise of $64 \%$. Turkey has few transactions for extracting safe conclusions and Spain does not have a competitive advantage (Table 3 and Figure 3).

\begin{tabular}{|c|c|c|c|c|c|c|c|}
\hline Year & Greece & Spain & France & Turkey & Netherlands & Croatia & Malta \\
\hline 2000 & 6,80 & 0,96 & - & - & - & 0,64 & - \\
\hline 2001 & 3,63 & 0,91 & 1,26 & - & - & - & - \\
\hline 2002 & 4,17 & 0,90 & - & - & - & - & - \\
\hline 2003 & 4,76 & 0,79 & 0,07 & - & - & - & - \\
\hline 2004 & 5,10 & 0,99 & 0,06 & - & - & - & - \\
\hline 2005 & 4,70 & 0,92 & 0,17 & - & - & 0,03 & - \\
\hline 2006 & 4,80 & 0,93 & 0,01 & - & - & 0,12 & - \\
\hline 2007 & 3,78 & 0,93 & 3,96 & - & - & - & - \\
\hline 2008 & 2,54 & 1,03 & 3,23 & - & - & - & - \\
\hline 2009 & 2,76 & 0,98 & 0,34 & 4,43 & - & - & - \\
\hline 2010 & 2,53 & 1,09 & 0,22 & - & - & - & - \\
\hline 2011 & 3,02 & 0,99 & 0,91 & 10,09 & - & - & - \\
\hline 2012 & 3,36 & 0,92 & 2,05 & 7,63 & - & - & - \\
\hline 2013 & 4,18 & 0,90 & 1,10 & 4,57 & - & 0,02 & - \\
\hline Max 2000-2007 & 6,80 & 0,99 & 3,96 & - & - & 0,64 & - \\
\hline Min 2000-2007 & 3,63 & 0,79 & 0,01 & - & - & 0,03 & - \\
\hline Average $2000-2007$ & 5,21 & 0,89 & 1,99 & - & - & 0,33 & - \\
\hline $\begin{array}{l}\text { Variation \% 2000- } \\
2007\end{array}$ & $-44,44$ & $-3,51$ & - & - & - & - & - \\
\hline $\begin{array}{l}\text { Std. Deviation } \\
2000-2007\end{array}$ & 0,99 & 0,06 & 1,56 & - & - & 0,33 & - \\
\hline Max 2008-2013 & 4,18 & 1,09 & 3,23 & 10,09 & - & 0,02 & - \\
\hline Min 2008-2013 & 2,53 & 0,90 & 0,22 & 4,43 & - & 0,02 & - \\
\hline $\begin{array}{l}\text { Average 2008- } \\
2013\end{array}$ & 3,35 & 0,99 & 1,72 & 7,26 & - & 0,02 & - \\
\hline $\begin{array}{l}\text { Variation \% 2008- } \\
2013\end{array}$ & 64,40 & $-12,60$ & $-66,00$ & - & - & - & - \\
\hline $\begin{array}{l}\text { Std. Deviation } \\
2008-2013\end{array}$ & 0,63 & 0,07 & 1,15 & 2,71 & - & - & - \\
\hline
\end{tabular}

Table 3: RXCA index-Market of Portugal.

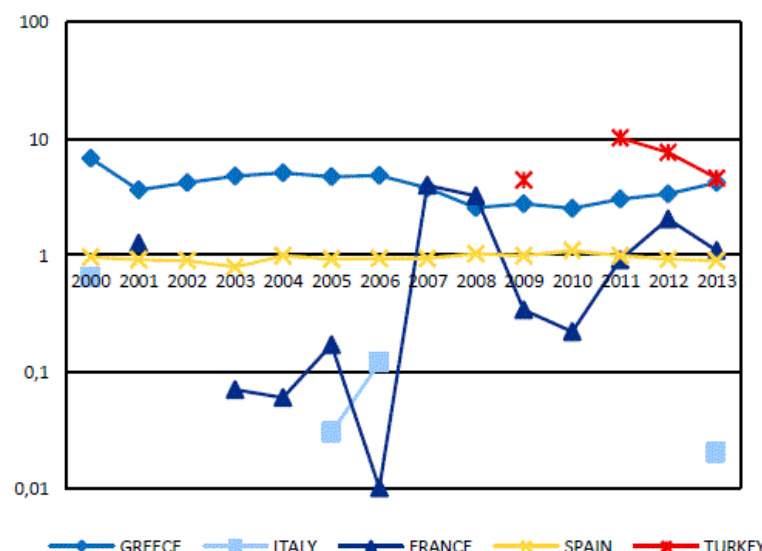

Figure 3: RXCA index-Market of Portugal 
Citation: Oikonomou A, Polymeros K (2015) Analyzing the Competitiveness of the Greek Sea Bream Exports in the European Union Market. J Glob Econ 3: 145. doi:10.4172/2375-4389.1000145

Page 5 of 10

Regarding the Spanish market, it was found that the Greek sea bream is first in import value share. The Greek RXCA index is kept firmly in the second place after Turkey for the last 6 years - from 2008 up to 2013 - at a level of 9 units and a decrease of $18 \%$. A significant upward change of Turkey's RXCA index is observed, at a rate of $12.66 \%$, with large fluctuations and a value of 47 units (Table 4 and Figure 4).

\begin{tabular}{|c|c|c|c|c|c|c|c|}
\hline Year & Greece & Italy & Spain & Turkey & Netherlands & Croatia & Malta \\
\hline 2000 & 9,93 & 0,31 & 0,61 & - & - & - & - \\
\hline 2001 & 3,93 & 0,35 & 1,14 & 32,08 & - & - & - \\
\hline 2002 & 2,35 & 3,30 & 0,57 & - & - & - & - \\
\hline 2003 & 4,61 & 0,13 & 0,48 & - & - & - & - \\
\hline 2004 & 3,12 & 0,14 & 0,64 & 148,95 & - & - & - \\
\hline 2005 & 2,93 & 0,13 & 0,37 & - & - & - & - \\
\hline 2006 & 6,99 & 0,05 & 0,13 & - & 0,47 & - & - \\
\hline 2007 & 5,70 & 0,38 & 0,87 & 27,93 & 0,20 & - & - \\
\hline 2008 & 9,62 & 0,30 & 0,56 & 16,67 & 0,05 & - & - \\
\hline 2009 & 10,16 & 0,08 & 0,27 & 3,09 & 0,02 & - & - \\
\hline 2010 & 9,89 & 0,04 & 0,20 & 2,76 & 0,01 & - & - \\
\hline 2011 & 10,03 & 0,03 & 0,18 & 11,87 & 0,01 & - & - \\
\hline 2012 & 7,90 & 0,02 & 0,89 & 91,78 & - & - & - \\
\hline 2013 & 7,91 & - & 0,39 & 18,78 & 0,02 & - & - \\
\hline Max 2000-2007 & 9,93 & 3,30 & 1,14 & 148,95 & 0,47 & - & - \\
\hline Min 2000-2007 & 2,35 & 0,05 & 0,13 & 27,93 & 0,20 & - & - \\
\hline Average $2000-2007$ & 6,14 & 1,68 & 0,63 & 88,44 & 0,33 & - & - \\
\hline $\begin{array}{l}\text { Variation \% 2000- } \\
2007\end{array}$ & $-42,55$ & 20,22 & 43,17 & - & - & - & - \\
\hline $\begin{array}{l}\text { Std. Deviation } \\
2000-2007\end{array}$ & 2,53 & 1,10 & 0,31 & 68,70 & 0,19 & - & - \\
\hline Max 2008-2013 & 10,16 & 0,30 & 0,89 & 91,78 & 0,05 & - & - \\
\hline Min 2008-2013 & 7,90 & 0,02 & 0,18 & 2,76 & 0,01 & - & - \\
\hline $\begin{array}{l}\text { Average 2008- } \\
2013\end{array}$ & 9,03 & 0,16 & 0,53 & 47,27 & 0,03 & - & - \\
\hline $\begin{array}{l}\text { Variation \% 2008- } \\
2013\end{array}$ & $-17,78$ & - & $-29,97$ & 12,66 & $-68,48$ & - & - \\
\hline $\begin{array}{l}\text { Std. Deviation } \\
2008-2013\end{array}$ & 1,06 & 0,12 & 0,27 & 33,80 & 0,02 & - & - \\
\hline
\end{tabular}

Table 4: RXCA index-Market of Spain.

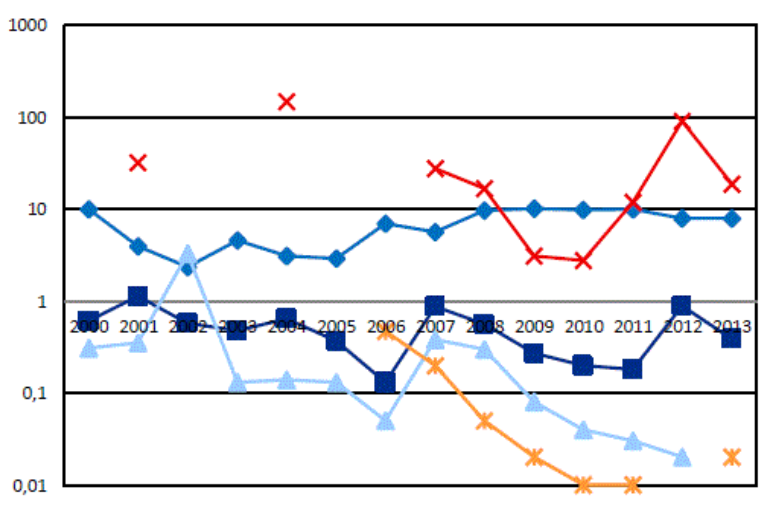

$\square$ GREECE $\longrightarrow$ FRANCE $\longrightarrow$ ITALY $\longrightarrow$ TURKEY $\longrightarrow$ NETHERLANDS

Figure 4: RXCA index-Market of Spain.
In the United Kingdom market, the Greek sea bream is first in import value share with an RXCA index at average 4 and a rise of $67 \%$. Turkey has large fluctuations of competitiveness index with a value of 7.5 units from 2009 onwards. The rest of the countries under the study have much lower competitive advantage, while Spain has no advantage (Table 5 and Figure 5).

\begin{tabular}{|l|l|l|l|l|l|l|l|}
\hline Year & Greece & Italy & Spain & Turkey & Netherlands & Croatia & Malta \\
\hline $\mathbf{2 0 0 0}$ & 7,39 & - & 2,17 & - & 0,07 & - & - \\
\hline $\mathbf{2 0 0 1}$ & 5,25 & - & 1,46 & - & 1,31 & - & - \\
\hline $\mathbf{2 0 0 2}$ & 4,36 & - & 1,69 & - & 0,28 & 0,13 & - \\
\hline $\mathbf{2 0 0 3}$ & 4,00 & - & 1,14 & - & 0,05 & - & - \\
\hline $\mathbf{2 0 0 4}$ & 3,68 & - & 1,48 & - & - & 0,09 & - \\
\hline $\mathbf{2 0 0 5}$ & 4,48 & 0,03 & 1,39 & - & 0,35 & 0,26 & - \\
\hline $\mathbf{2 0 0 6}$ & 4,69 & 0,11 & 1,67 & - & 0,08 & - & - \\
\hline $\mathbf{2 0 0 7}$ & 4,10 & 0,06 & 2,48 & - & 0,68 & 2,02 & - \\
\hline $\mathbf{2 0 0 8}$ & 3,19 & - & 1,86 & - & 1,91 & 1,75 & - \\
\hline $\mathbf{2 0 0 9}$ & 2,82 & 0,06 & 2,98 & 2,62 & 2,15 & 1,92 & - \\
\hline $\mathbf{2 0 1 0}$ & 3,08 & 0,21 & 2,00 & 7,53 & 2,03 & 2,15 & - \\
\hline $\mathbf{2 0 1 1}$ & 2,95 & 0,47 & 1,88 & 12,25 & 1,18 & 2,28 & - \\
\hline $\mathbf{2 0 1 2}$ & 3,11 & 0,22 & 1,69 & 5,64 & 1,24 & 1,07 & - \\
\hline $\mathbf{2 0 1 3}$ & 5,36 & 0,68 & 1,85 & 3,70 & 2,38 & 2,05 & - \\
\hline Max 2000-2007 & 7,39 & 0,11 & 2,48 & - & 1,31 & 2,02 & - \\
\hline Min 2000-2007 & 3,68 & 0,03 & 1,14 & - & 0,05 & 0,09 & - \\
\hline Average 2000- & 5,53 & 0,07 & 1,81 & - & 0,68 & 1,06 & - \\
\hline $\mathbf{2 0 0 7}$ & & & & & & & - \\
\hline Variation \% 2000- & $-44,45$ & - & 14,34 & - & 947,34 & - & - \\
\hline $\mathbf{2 0 0 7}$ & 1,17 & 0,04 & 0,44 & - & 0,46 & 0,93 & - \\
\hline $\begin{array}{l}\text { Std. Deviation } \\
\mathbf{2 0 0 0 - 2 0 0 7}\end{array}$ & & & & & & & \\
\hline Max 2008-2013 & 5,36 & 0,68 & 2,98 & 12,25 & 2,38 & 2,28 & - \\
\hline Min 2008-2013 & 2,82 & 0,06 & 1,69 & 2,62 & 1,18 & 1,07 & - \\
\hline Average 2008- & 4,09 & 0,37 & 2,33 & 7,43 & 1,78 & 1,68 & - \\
\hline $\mathbf{2 0 1 3}$ & & & & & & & \\
\hline Variation \% 2008- & 67,69 & - & $-0,46$ & - & 24,24 & 16,98 & - \\
\hline $\mathbf{2 0 1 3}$ & & & & & & & \\
\hline Std. Deviation & 0,96 & 0,25 & 0,47 & 3,79 & 0,49 & 0,43 & - \\
\hline $\mathbf{2 0 0 8 - 2 0 1 3}$ & & & & & & & \\
\hline
\end{tabular}

Table 5: RXCA index-Market of UK.

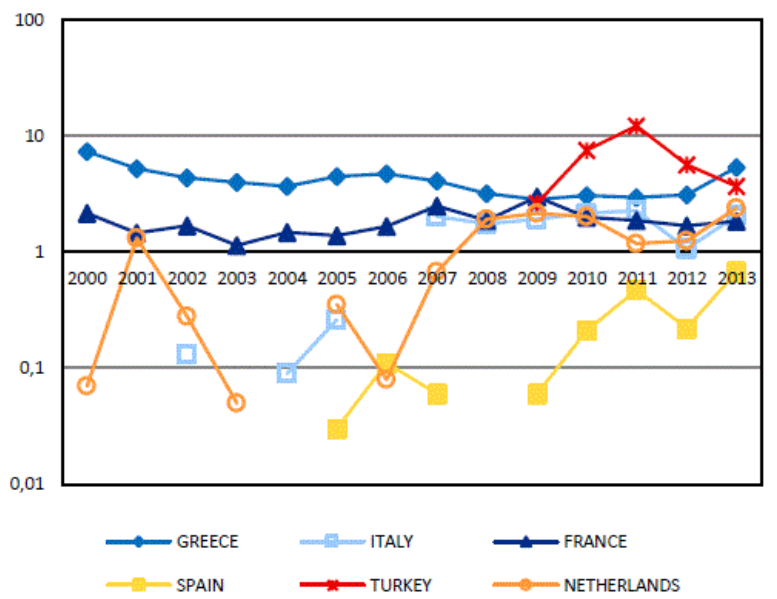

Figure 5: RXCA index-Market of UK. 
Citation: Oikonomou A, Polymeros K (2015) Analyzing the Competitiveness of the Greek Sea Bream Exports in the European Union Market. J Glob Econ 3: 145. doi:10.4172/2375-4389.1000145

Page 6 of 10

As for the German market, Greece ranks first in a competitive advantage on average 12 units but a decrease of $18 \%$. Turkey, with RXCA indexes at 7 units had a huge increase of $430 \%$. Italy and France have much lower competitive advantage (Table 6 and Figure 6).

\begin{tabular}{|c|c|c|c|c|c|c|c|}
\hline Year & Greece & Italy & Spain & Turkey & Netherlands & Croatia & Malta \\
\hline 2000 & 13,83 & 11,63 & 3,29 & - & 0,19 & 1,41 & - \\
\hline 2001 & 15,31 & 11,60 & 2,31 & - & 0,19 & 0,23 & - \\
\hline 2002 & 16,76 & 11,51 & 1,03 & - & 0,18 & 0,11 & - \\
\hline 2003 & 10,37 & 13,31 & 1,14 & 0,91 & 0,12 & 0,28 & - \\
\hline 2004 & 10,90 & 16,70 & 1,36 & 29,78 & 0,17 & 0,19 & - \\
\hline 2005 & 9,73 & 10,26 & 1,05 & 16,96 & 0,22 & 0,14 & - \\
\hline 2006 & 8,93 & 10,50 & 1,26 & - & 0,21 & 0,38 & - \\
\hline 2007 & 13,67 & 9,44 & 1,84 & - & 0,25 & 2,13 & - \\
\hline 2008 & 12,90 & 6,91 & 1,24 & 1,99 & 0,24 & 0,76 & - \\
\hline 2009 & 11,27 & 5,62 & 1,97 & 6,65 & 0,31 & 0,98 & - \\
\hline 2010 & 11,36 & 5,30 & 1,64 & 12,51 & 0,35 & 0,53 & - \\
\hline 2011 & 12,24 & 5,11 & 1,64 & 8,49 & 0,34 & 0,95 & - \\
\hline 2012 & 11,45 & 2,46 & 1,07 & 10,55 & 0,55 & 0,86 & - \\
\hline 2013 & 10,53 & 5,95 & 0,99 & 10,62 & 1,32 & 0,23 & - \\
\hline Max 2000-2007 & 16,76 & 16,70 & 3,29 & 29,78 & 0,25 & 2,13 & - \\
\hline Min 2000-2007 & 8,93 & 9,44 & 1,03 & 0,91 & 0,12 & 0,11 & - \\
\hline $\begin{array}{l}\text { Average 2000- } \\
2007\end{array}$ & 12,84 & 13,07 & 2,16 & 15,35 & 0,18 & 1,12 & - \\
\hline $\begin{array}{l}\text { Variation \% } \\
2000-2007\end{array}$ & $-1,10$ & $-18,85$ & $-44,05$ & - & 29,82 & 50,56 & - \\
\hline $\begin{array}{l}\text { Std. Deviation } \\
2000-2007\end{array}$ & 2,84 & 2,27 & 0,79 & 14,46 & 0,04 & 0,75 & - \\
\hline Max 2008-2013 & 12,90 & 6,91 & 1,97 & 12,51 & 1,32 & 0,98 & - \\
\hline Min 2008-2013 & 10,53 & 2,46 & 0,99 & 1,99 & 0,24 & 0,23 & - \\
\hline $\begin{array}{l}\text { Average 2008- } \\
2013\end{array}$ & 11,71 & 4,69 & 1,48 & 7,25 & 0,78 & 0,61 & - \\
\hline $\begin{array}{l}\text { Variation \% } \\
2008-2013\end{array}$ & $-18,35$ & $-13,99$ & $-20,20$ & 433,29 & 445,88 & $-69,61$ & - \\
\hline $\begin{array}{l}\text { Std. Deviation } \\
2008-2013\end{array}$ & 0,83 & 1,50 & 0,39 & 3,76 & 0,41 & 0,29 & - \\
\hline
\end{tabular}

Table 6: RXCA index-Market of Germany.

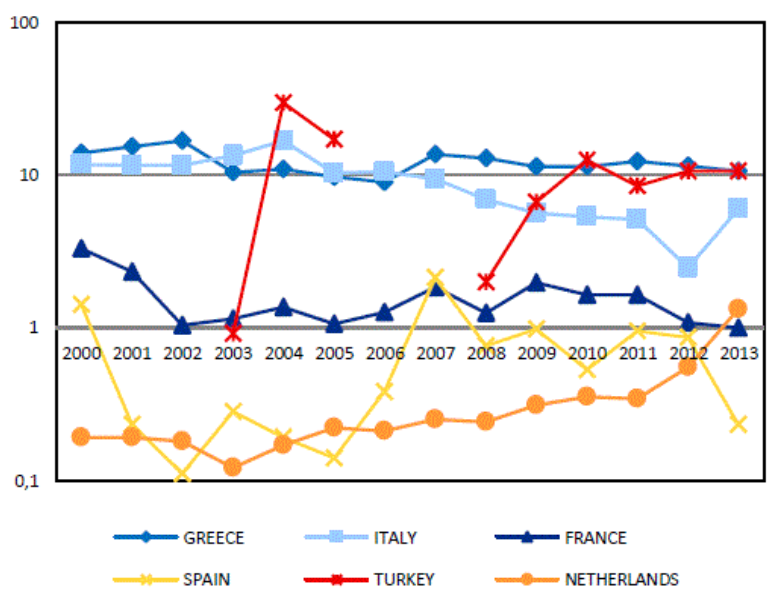

Figure 6: RXCA index-Market of Germany.
Concerning the Romanian market, which is a growing market for the Greek sea bream, the last 6 years from 2008 up to 2013, it was found that the latter has won first place with a steady course and a competitiveness index at 5 units, followed by Turkey with an index of 4 units (Table 7 and Figure 7).

\begin{tabular}{|c|c|c|c|c|c|c|c|}
\hline Year & Greece & Spain & France & Turkey & Netherlands & Italy & Malta \\
\hline 2000 & - & 32,26 & - & 109,22 & - & - & - \\
\hline 2001 & 1,88 & - & 12,28 & 28,30 & - & - & - \\
\hline 2002 & 0,18 & - & 9,07 & 7,35 & - & - & - \\
\hline 2003 & 2,15 & - & 11,49 & - & - & - & - \\
\hline 2004 & 3,09 & - & 1,33 & - & - & - & - \\
\hline 2005 & 3,45 & - & 0,45 & - & 0,42 & 0,07 & - \\
\hline 2006 & 2,90 & - & 0,13 & - & 1,18 & 0,04 & - \\
\hline 2007 & 7,48 & - & 0,44 & - & 0,08 & 0,35 & - \\
\hline 2008 & 5,52 & - & 0,64 & - & 0,17 & 1,59 & - \\
\hline 2009 & 6,00 & - & 0,02 & 4,76 & - & 0,86 & - \\
\hline 2010 & 5,71 & - & 0,03 & 3,78 & - & 0,20 & - \\
\hline 2011 & 5,04 & - & 0,08 & 4,29 & - & 0,26 & - \\
\hline 2012 & 4,58 & - & - & 4,00 & - & 0,09 & - \\
\hline 2013 & 5,33 & - & 0,22 & 4,83 & - & 0,42 & - \\
\hline Max 2000-2007 & 7,48 & - & 12,28 & 109,22 & 1,18 & 0,35 & - \\
\hline Min 2000-2007 & 0,18 & - & 0,13 & 7,35 & 0,08 & 0,04 & - \\
\hline $\begin{array}{l}\text { Average 2000- } \\
2007\end{array}$ & 3,83 & - & 6,20 & 58,29 & 0,63 & 0,20 & - \\
\hline $\begin{array}{l}\text { Variation \% } \\
2000-2007\end{array}$ & 298,20 & - & $-96,41$ & - & - & - & - \\
\hline $\begin{array}{l}\text { Std. Deviation } \\
2000-2007\end{array}$ & 2,24 & - & 5,63 & 53,80 & 0,57 & 0,17 & - \\
\hline Max 2008-2013 & 6,00 & - & 0,64 & 4,83 & - & 1,59 & - \\
\hline Min 2008-2013 & 4,58 & - & 0,02 & 3,78 & - & 0,09 & - \\
\hline $\begin{array}{l}\text { Average 2008- } \\
2013\end{array}$ & 5,29 & - & 0,33 & 4,31 & - & 0,84 & - \\
\hline $\begin{array}{l}\text { Variation \% } \\
2008-2013\end{array}$ & $-3,59$ & - & $-65,29$ & - & - & $-73,81$ & - \\
\hline $\begin{array}{l}\text { Std. Deviation } \\
2008-2013\end{array}$ & 0,51 & - & 0,26 & 0,46 & - & 0,57 & - \\
\hline
\end{tabular}

Table 7: RXCA index values-Market of Romania.
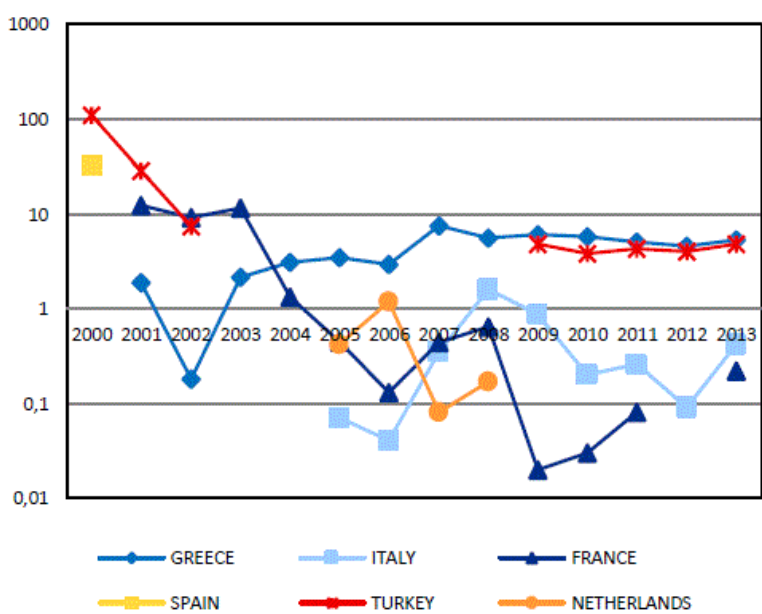

Figure 7: RXCA index-Market of Romania. 
Citation: Oikonomou A, Polymeros K (2015) Analyzing the Competitiveness of the Greek Sea Bream Exports in the European Union Market. J Glob Econ 3: 145. doi:10.4172/2375-4389.1000145

Page 7 of 10

Obviously, values of RXCA index show sharp changes, suggesting that the sea bream market is characterized by a highly competitive and rapidly changing environment, creating opportunities and threats to all involved branches. Threats from the new entry exporting countries, in existing or emerging markets, such as Turkey. And finally opportunities by the opening of new import markets, such as the Romanian market.

Concerning the selling prices of sea bream among the competitive countries in the Italian market shows that the products of Turkey and Malta have on average the lowest prices, with regard to Spain and France and third lowest price the Greek's one. Croatia appears as a new competitor. However, in the last 6 years, from 2008 up to 2013, almost all prices are rising (Table 8 ).

In French, Portugal and Spanish markets, it is found that the product of Greece and Turkey has the lowest prices. The Greek sea bream is the cheapest in France and Portugal, whereas transactions of Turkey with these countries are too few to be conclusive regarding the prices (Tables 9-11).

In the United Kingdom market, it seems that the Greek product has the lowest prices. In Romania, it was found that the products of Greece and Turkey show the lowest prices, with a relatively little difference between them (Tables 12 and 13).

Finally, based on calculations of sea bream's selling prices in the German market, Turkey has the lowest price, followed by Greece, while till 2007; the Greek sea bream was the cheapest (Table 14).

\begin{tabular}{|c|c|c|c|c|c|c|c|}
\hline Year & Greece & Spain & France & Turkey & Netherlands & Croatia & Malta \\
\hline 2000 & 4,51 & 8,99 & 4,76 & 4,12 & 5,06 & 3,91 & 3,12 \\
\hline 2001 & 3,70 & 7,99 & 5,89 & 3,56 & 4,73 & 3,27 & 2,82 \\
\hline 2002 & 3,64 & 8,30 & 6,99 & 3,28 & 4,30 & 3,47 & 2,62 \\
\hline 2003 & 3,69 & 8,85 & 6,98 & 3,37 & 3,31 & 4,10 & 3,79 \\
\hline 2004 & 4,31 & 8,94 & 6,55 & 4,45 & 4,21 & 4,25 & 5,07 \\
\hline 2005 & 4,25 & 7,39 & 6,45 & 3,64 & 7,77 & 4,53 & 4,79 \\
\hline 2006 & 4,30 & 7,73 & 7,18 & 3,58 & 6,73 & 4,37 & 4,74 \\
\hline 2007 & 4,03 & 7,88 & 8,41 & 3,44 & 7,35 & 4,85 & 4,15 \\
\hline 2008 & 3,37 & 8,69 & 8,67 & 2,84 & 7,09 & 4,53 & 3,26 \\
\hline 2009 & 3,76 & 9,55 & 7,16 & 3,18 & 6,89 & 4,30 & 3,45 \\
\hline 2010 & 4,30 & 10,51 & 9,32 & 3,74 & 7,67 & 4,49 & 4,01 \\
\hline 2011 & 5,02 & 10,27 & 9,06 & 4,69 & 8,28 & 4,97 & 4,86 \\
\hline 2012 & 4,18 & 10,77 & 9,06 & 3,53 & 6,71 & 4,61 & 4,09 \\
\hline 2013 & 4,13 & 11,11 & 10,54 & 3,86 & 6,02 & 4,71 & 4,23 \\
\hline Max 2000-2007 & 4,51 & 8,99 & 8,41 & 4,45 & 7,77 & 4,85 & 5,07 \\
\hline Min 2000-2007 & 3,64 & 7,39 & 4,76 & 3,28 & 3,31 & 3,27 & 2,62 \\
\hline $\begin{array}{l}\text { Average 2000- } \\
2007\end{array}$ & 4,07 & 8,19 & 6,59 & 3,86 & 5,54 & 4,06 & 3,85 \\
\hline $\begin{array}{l}\text { Variation \% } \\
2000-2007\end{array}$ & $-10,70$ & $-12,32$ & 76,57 & $-16,60$ & 45,31 & 24,10 & 32,95 \\
\hline $\begin{array}{l}\text { Std. Deviation } \\
2000-2007\end{array}$ & 5,02 & 11,11 & 10,54 & 4,69 & 8,28 & 4,97 & 4,86 \\
\hline Max 2008-2013 & 3,37 & 8,69 & 7,16 & 2,84 & 6,02 & 4,30 & 3,26 \\
\hline Min 2008-2013 & 4,19 & 9,90 & 8,85 & 3,76 & 7,15 & 4,63 & 4,06 \\
\hline $\begin{array}{l}\text { Average 2008- } \\
2013\end{array}$ & 5,02 & 11,11 & 10,54 & 4,69 & 8,28 & 4,97 & 4,86 \\
\hline $\begin{array}{l}\text { Variation \% } \\
\text { 2008-2013 }\end{array}$ & $-3,59$ & - & $-65,29$ & - & - & $-73,81$ & - \\
\hline $\begin{array}{l}\text { Std. Deviation } \\
\text { 2008-2013 }\end{array}$ & 0,51 & - & 0,26 & 0,46 & - & 0,57 & - \\
\hline
\end{tabular}

Table 8: Selling Prices-Market of Italy.

\begin{tabular}{|l|c|c|c|c|c|c|c|}
\hline Year & Greece & Italy & Spain & Turkey & Netherlands & Croatia & Malta \\
\hline $\mathbf{2 0 0 0}$ & 5,39 & 6,71 & 5,73 & - & 5,88 & - & - \\
\hline $\mathbf{2 0 0 1}$ & 3,32 & 5,53 & 5,59 & - & 4,44 & - & - \\
\hline $\mathbf{2 0 0 2}$ & 3,43 & 5,24 & 5,95 & 4,57 & 5,37 & - & - \\
\hline $\mathbf{2 0 0 3}$ & 4,07 & 4,70 & 5,77 & - & 3,96 & - & - \\
\hline $\mathbf{2 0 0 4}$ & 3,89 & 4,47 & 5,62 & - & 4,81 & - & - \\
\hline $\mathbf{2 0 0 5}$ & 3,62 & 4,80 & 5,09 & - & 7,27 & - & - \\
\hline $\mathbf{2 0 0 6}$ & 4,09 & 5,24 & 5,00 & - & 6,54 & - & - \\
\hline $\mathbf{2 0 0 7}$ & 4,28 & 8,08 & 5,38 & - & 4,24 & - & - \\
\hline $\mathbf{2 0 0 8}$ & 3,32 & 7,33 & 4,34 & 2,92 & 3,47 & - & - \\
\hline $\mathbf{2 0 0 9}$ & 3,63 & 5,01 & 3,92 & & 4,37 & - & - \\
\hline $\mathbf{2 0 1 0}$ & 4,14 & 4,79 & 4,49 & 4,14 & 4,52 & - & - \\
\hline $\mathbf{2 0 1 1}$ & 5,14 & 5,73 & 5,19 & - & 5,95 & - & - \\
\hline $\mathbf{2 0 1 2}$ & 4,71 & 4,23 & 5,20 & - & 4,61 & - & - \\
\hline $\mathbf{2 0 1 3}$ & 3,57 & 4,64 & 4,84 & 3,99 & 4,25 & - & - \\
\hline Max 2000-2007 & 5,39 & 8,08 & 5,95 & 4,57 & 7,27 & - & - \\
\hline Min 2000-2007 & 3,32 & 4,47 & 5,00 & 4,57 & 3,96 & - & - \\
\hline Average 2000- & 4,35 & 6,27 & 5,48 & 4,57 & 5,62 & - & - \\
\hline $\mathbf{2 0 0 7}$ & & & & & $-27,87$ & - & - \\
\hline Variation \% & $-20,50$ & 20,48 & $-6,08$ & - & & & - \\
\hline $\mathbf{2 0 0 0 - 2 0 0 7}$ & & & & & 5,95 & - & - \\
\hline Max 2008-2013 & 5,14 & 7,33 & 5,20 & 4,14 & 5,47 & - & - \\
\hline Min 2008-2013 & 3,32 & 4,23 & 3,92 & 2,92 & 3,47 & - & - \\
\hline Average 2008- & 4,23 & 5,78 & 4,56 & 3,53 & 4,71 & - & \\
\hline $\mathbf{2 0 1 3}$ & & & & & & & - \\
\hline Variation \% & 7,49 & $-36,64$ & 11,40 & 36,54 & 22,68 & - & - \\
\hline $\mathbf{2 0 0 8 - 2 0 1 3}$ & & & & & & & \\
\hline
\end{tabular}

Table 9: Selling Prices-Market of France.

\begin{tabular}{|l|c|c|c|c|c|c|c|}
\hline Year & Greece & Spain & France & Turkey & Netherlands & Italy & Malta \\
\hline $\mathbf{2 0 0 0}$ & 5,39 & 6,71 & - & - & - & 4,58 & - \\
\hline $\mathbf{2 0 0 1}$ & 3,32 & 5,53 & 4,09 & - & - & - & - \\
\hline $\mathbf{2 0 0 2}$ & 3,43 & 5,24 & - & - & - & - & - \\
\hline $\mathbf{2 0 0 3}$ & 4,07 & 4,70 & 6,62 & - & - & - & - \\
\hline $\mathbf{2 0 0 4}$ & 3,89 & 4,47 & 5,89 & - & - & - & - \\
\hline $\mathbf{2 0 0 5}$ & 3,62 & 4,80 & 4,80 & - & - & 3,94 & - \\
\hline $\mathbf{2 0 0 6}$ & 4,09 & 5,24 & 17,64 & - & - & 4,27 & - \\
\hline $\mathbf{2 0 0 7}$ & 4,28 & 8,08 & 3,84 & - & - & - & - \\
\hline $\mathbf{2 0 0 8}$ & 3,32 & 7,33 & 3,91 & - & - & - & - \\
\hline $\mathbf{2 0 0 9}$ & 3,63 & 5,01 & 8,85 & 2,91 & - & - & - \\
\hline $\mathbf{2 0 1 0}$ & 4,14 & 4,79 & 10,42 & - & - & - & - \\
\hline $\mathbf{2 0 1 1}$ & 5,14 & 5,73 & 6,77 & 3,51 & - & - & - \\
\hline $\mathbf{2 0 1 2}$ & 4,71 & 4,23 & 5,93 & 3,79 & - & - & - \\
\hline $\mathbf{2 0 1 3}$ & 3,57 & 4,64 & 4,72 & 3,38 & - & 3,79 & - \\
\hline Max 2000-2007 & 5,39 & 8,08 & 17,64 & - & - & 4,58 & - \\
\hline Min 2000-2007 & 3,32 & 4,47 & 3,84 & - & - & 3,94 & - \\
\hline Average 2000- & 4,35 & 6,27 & 10,74 & - & - & 4,26 & - \\
\hline $\mathbf{2 0 0 7}$ & & & & & & - & - \\
\hline Variation \% & $-20,50$ & 20,48 & - & - & - & - & - \\
\hline $\mathbf{2 0 0 0 - 2 0 0 7}$ & & & & & & 3,79 & - \\
\hline Max 2008-2013 & 5,14 & 7,33 & 10,42 & 3,79 & - & 3,79 & - \\
\hline Min 2008-2013 & 3,32 & 4,23 & 3,91 & 2,91 & - & - & - \\
\hline Average 2008- & 4,23 & 5,78 & 7,16 & 3,53 & - & - & - \\
\hline $\mathbf{2 0 1 3}$ & & & & & & - & - \\
\hline Variation \% & 7,49 & $-36,64$ & 20,80 & - & - & - & - \\
\hline $\mathbf{2 0 0 8 - 2 0 1 3}$ & & & & & & - & - \\
\hline
\end{tabular}

Table 10: Selling Prices-Market of Portugal 
Citation: Oikonomou A, Polymeros K (2015) Analyzing the Competitiveness of the Greek Sea Bream Exports in the European Union Market. J Glob Econ 3: 145. doi:10.4172/2375-4389.1000145

Page 8 of 10

\begin{tabular}{|l|c|c|c|c|c|c|c|}
\hline Year & Greece & Spain & France & Turkey & Netherlands & Italy & Malta \\
\hline $\mathbf{2 0 0 0}$ & 4,84 & 1,22 & 1,94 & - & - & - & - \\
\hline $\mathbf{2 0 0 1}$ & 4,57 & 50,21 & 1,61 & 3,41 & - & - & - \\
\hline $\mathbf{2 0 0 2}$ & 3,83 & 2,33 & 2,11 & - & - & - & - \\
\hline $\mathbf{2 0 0 3}$ & 3,89 & 1,40 & 3,92 & - & - & - & - \\
\hline $\mathbf{2 0 0 4}$ & 4,69 & 1,27 & 8,84 & 4,28 & 2,33 & - & - \\
\hline $\mathbf{2 0 0 5}$ & 4,63 & 3,21 & 15,63 & - & - & - & - \\
\hline $\mathbf{2 0 0 6}$ & 5,07 & 1,98 & 7,42 & - & 6,61 & - & - \\
\hline $\mathbf{2 0 0 7}$ & 3,97 & 2,01 & 0,78 & 3,62 & 4,07 & - & - \\
\hline $\mathbf{2 0 0 8}$ & 3,43 & 2,33 & 39,34 & 3,13 & 5,22 & - & - \\
\hline $\mathbf{2 0 0 9}$ & 3,67 & 3,87 & 25,54 & 3,46 & 6,45 & - & - \\
\hline $\mathbf{2 0 1 0}$ & 4,22 & 1,77 & 27,07 & 3,90 & 4,97 & - & - \\
\hline $\mathbf{2 0 1 1}$ & 5,29 & 3,80 & 17,31 & 4,78 & 6,41 & - & - \\
\hline $\mathbf{2 0 1 2}$ & 4,95 & 4,01 & 21,59 & 3,91 & - & - & - \\
\hline $\mathbf{2 0 1 3}$ & 5,14 & - & 21,99 & 3,86 & 1,44 & - & - \\
\hline Max 2000-2007 & 5,07 & 50,21 & 15,63 & 4,28 & 6,61 & - & - \\
\hline Min 2000-2007 & 3,83 & 1,22 & 0,78 & 3,41 & 2,33 & - & - \\
\hline Average 2000- & 4,45 & 25,72 & 8,20 & 3,84 & 4,47 & - & - \\
\hline $\mathbf{2 0 0 7}$ & & & & & & & \\
\hline $\begin{array}{l}\text { Variation \% } \\
\text { 2000-2007 }\end{array}$ & $-18,02$ & 64,44 & $-59,81$ & - & - & - & - \\
\hline Max 2008-2013 & 5,29 & 4,01 & 39,34 & 4,78 & 6,45 & - & - \\
\hline Min 2008-2013 & 3,43 & 1,77 & 17,31 & 3,13 & 1,44 & - & - \\
\hline Average 2008- & 4,36 & 2,89 & 28,33 & 3,95 & 3,95 & - & - \\
\hline $\mathbf{2 0 1 3}$ & 49,60 & - & $-44,11$ & 23,53 & $-72,36$ & - & - \\
\hline Variation \% & 49613 & & & & & & \\
\hline $\mathbf{2 0 0 8 - 2 0 1 3}$ & & & & & - \\
\hline
\end{tabular}

Table 11: Selling Prices-Market of Spain.

\begin{tabular}{|l|c|c|c|c|c|c|c|}
\hline Year & Greece & Spain & France & Turkey & Netherlands & Italy & Malta \\
\hline $\mathbf{2 0 0 0}$ & 6,81 & - & 7,21 & - & 5,29 & - & - \\
\hline $\mathbf{2 0 0 1}$ & 5,61 & - & 5,74 & - & 5,60 & - & - \\
\hline $\mathbf{2 0 0 2}$ & 5,04 & - & 5,79 & - & 4,92 & 5,62 & - \\
\hline $\mathbf{2 0 0 3}$ & 4,58 & 1,65 & 4,76 & - & 4,78 & - & - \\
\hline $\mathbf{2 0 0 4}$ & 5,30 & - & 5,86 & - & - & 6,75 & - \\
\hline $\mathbf{2 0 0 5}$ & 4,50 & 4,75 & 5,60 & - & 4,85 & 4,19 & - \\
\hline $\mathbf{2 0 0 6}$ & 4,81 & 4,80 & 5,89 & - & 4,05 & - & - \\
\hline $\mathbf{2 0 0 7}$ & 5,01 & 4,49 & 5,14 & - & 3,84 & 3,18 & - \\
\hline $\mathbf{2 0 0 8}$ & 2,50 & - & 3,98 & - & 3,48 & 2,78 & - \\
\hline $\mathbf{2 0 0 9}$ & 3,84 & 4,70 & 4,04 & 3,59 & 3,66 & 3,32 & - \\
\hline $\mathbf{2 0 1 0}$ & 4,42 & 4,41 & 4,42 & 3,84 & 4,56 & 3,97 & - \\
\hline $\mathbf{2 0 1 1}$ & 4,58 & 5,85 & 5,58 & 4,16 & 5,18 & 4,65 & - \\
\hline $\mathbf{2 0 1 2}$ & 2,56 & 5,23 & 6,76 & 4,54 & 4,08 & 3,64 & - \\
\hline $\mathbf{2 0 1 3}$ & 2,41 & 4,44 & 4,45 & 5,31 & 4,08 & 4,11 & - \\
\hline Max 2000-2007 & 6,81 & 4,80 & 7,21 & 0,00 & 5,60 & 6,75 & - \\
\hline Min 2000-2007 & 4,50 & 1,65 & 4,76 & 0,00 & 3,84 & 3,18 & - \\
\hline Average 2000- & 5,66 & 3,22 & 5,99 & 0,00 & 4,72 & 4,96 & - \\
\hline $\mathbf{2 0 0 7}$ & & & & & & & \\
\hline $\begin{array}{l}\text { Variation \% 2000- } \\
\text { 2007 }\end{array}$ & $-26,47$ & - & $-28,69$ & - & $-27,38$ & - & - \\
\hline Max 2008-2013 & 4,58 & 5,85 & 6,76 & 5,31 & 5,18 & 4,65 & - \\
\hline Min 2008-2013 & 2,41 & 4,41 & 3,98 & 3,59 & 3,48 & 2,78 & - \\
\hline Average 2008- & 3,50 & 5,13 & 5,37 & 4,45 & 4,33 & 3,71 & - \\
\hline $\mathbf{2 0 1 3}$ & & & & & & & \\
\hline Variation \% 2008- & $-3,65$ & - & 11,79 & - & 17,43 & 47,73 & - \\
\hline $\mathbf{2 0 1 3}$ & & & & & & & \\
\hline
\end{tabular}

Table 12: Selling Prices-Market of UK.

\begin{tabular}{|l|c|c|c|c|c|c|c|c|}
\hline Year & Greece & Spain & France & Turkey & Netherlands & Italy & Malta \\
\hline $\mathbf{2 0 0 0}$ & - & - & - & 5,34 & - & - & - \\
\hline $\mathbf{2 0 0 1}$ & 9,15 & - & 9,07 & 5,61 & - & - & - \\
\hline $\mathbf{2 0 0 2}$ & - & - & 8,20 & 3,57 & - & - & - \\
\hline $\mathbf{2 0 0 3}$ & 4,82 & - & 7,55 & - & - & - & - \\
\hline $\mathbf{2 0 0 4}$ & 6,79 & - & 8,75 & - & - & - & - \\
\hline $\mathbf{2 0 0 5}$ & 4,66 & - & 11,04 & - & 4,71 & 8,20 & - \\
\hline $\mathbf{2 0 0 6}$ & 4,77 & - & 12,15 & - & 7,54 & 5,03 & - \\
\hline $\mathbf{2 0 0 7}$ & 4,30 & - & 5,39 & - & 12,96 & 4,13 & - \\
\hline $\mathbf{2 0 0 8}$ & 3,67 & - & 3,68 & - & 8,19 & 3,64 & - \\
\hline $\mathbf{2 0 0 9}$ & 4,25 & - & - & 3,38 & - & 3,69 & - \\
\hline $\mathbf{2 0 1 0}$ & 4,68 & - & - & 3,92 & - & 4,46 & - \\
\hline $\mathbf{2 0 1 1}$ & 5,65 & - & - & 4,84 & - & 5,79 & - \\
\hline $\mathbf{2 0 1 2}$ & 4,55 & - & - & 4,07 & - & 7,42 & - \\
\hline $\mathbf{2 0 1 3}$ & 4,33 & - & 5,39 & 3,96 & - & 5,33 & - \\
\hline Max 2000-2007 & 9,15 & - & 12,15 & 5,61 & 12,96 & 8,20 & - \\
\hline Min 2000-2007 & 4,30 & 0,00 & 5,39 & 3,57 & 4,71 & 4,13 & - \\
\hline Average 2000- & 6,72 & - & 8,77 & 4,59 & 8,84 & 6,17 & - \\
\hline $\mathbf{2 0 0 7}$ & & & & & & & \\
\hline Variation \% 2000- & $-53,03$ & - & $-40,62$ & - & - & - & - \\
\hline $\mathbf{2 0 0 7}$ & & & & & & & \\
\hline Max 2008-2013 & 5,65 & 0,00 & 5,39 & 4,84 & 8,19 & 7,42 & - \\
\hline Min 2008-2013 & 3,67 & 0,00 & 3,68 & 3,38 & 8,19 & 3,64 & - \\
\hline Average 2008- & 4,66 & 0,00 & 4,54 & 4,11 & 8,19 & 5,53 & - \\
\hline $\mathbf{2 0 1 3}$ & & & & & & & \\
\hline Variation \% 2008- & 17,85 & - & 46,47 & - & - & 46,63 & - \\
\hline $\mathbf{2 0 1 3}$ & & & & & & & \\
\hline
\end{tabular}

2013

Table 13: Selling Prices-Market of Romania.

\begin{tabular}{|l|c|c|c|c|c|c|c|c|}
\hline Year & Greec & Italy & France & Turkey & Netherlands & Spain & Malta \\
\hline $\mathbf{2 0 0 0}$ & 5,82 & 6,35 & 8,34 & - & 4,71 & & - \\
\hline $\mathbf{2 0 0 1}$ & 5,15 & 6,19 & 7,35 & - & 5,43 & 5,67 & - \\
\hline $\mathbf{2 0 0 2}$ & 4,33 & 5,62 & 6,82 & - & 5,74 & 4,35 & - \\
\hline $\mathbf{2 0 0 3}$ & 4,15 & 5,61 & 6,04 & 5,95 & 5,15 & 5,67 & - \\
\hline $\mathbf{2 0 0 4}$ & 4,83 & 5,93 & 6,80 & 5,89 & 5,06 & 5,78 & - \\
\hline $\mathbf{2 0 0 5}$ & 4,53 & 5,85 & 6,68 & 4,41 & 5,24 & 5,36 & - \\
\hline $\mathbf{2 0 0 6}$ & 4,91 & 6,23 & 6,67 & - & 5,65 & 5,73 & - \\
\hline $\mathbf{2 0 0 7}$ & 4,50 & 5,37 & 6,77 & - & 5,65 & 6,76 & - \\
\hline $\mathbf{2 0 0 8}$ & 3,71 & 4,55 & 6,56 & 2,64 & 5,39 & 6,05 & - \\
\hline $\mathbf{2 0 0 9}$ & 4,17 & 4,85 & 3,72 & 4,06 & 5,42 & 5,58 & - \\
\hline $\mathbf{2 0 1 0}$ & 4,67 & 5,49 & 6,37 & 4,58 & 5,71 & 6,77 & - \\
\hline $\mathbf{2 0 1 1}$ & 5,80 & 6,30 & 8,38 & 4,96 & 7,20 & 7,48 & - \\
\hline $\mathbf{2 0 1 2}$ & 5,21 & 5,46 & 8,50 & 3,43 & 4,43 & 7,13 & - \\
\hline $\mathbf{2 0 1 3}$ & 5,66 & 5,99 & 8,21 & 3,70 & 4,34 & 6,59 & - \\
\hline Max 2000-2007 & 5,82 & 6,35 & 8,34 & 5,95 & 5,74 & 8,40 & - \\
\hline Min 2000-2007 & 4,15 & 5,37 & 6,04 & 4,41 & 4,71 & 4,35 & - \\
\hline Average 2000-2007 & 4,99 & 5,86 & 7,19 & 5,18 & 5,23 & 6,38 & - \\
\hline Variation \% 2000- & $-22,69$ & $-15,42$ & $-18,84$ & - & 19,75 & $-19,56$ & - \\
\hline $\mathbf{2 0 0 7}$ & & & & & & & \\
\hline Max 2008-2013 & 5,80 & 6,30 & 8,50 & 4,96 & 7,20 & 7,48 & - \\
\hline Min 2008-2013 & 3,71 & 4,55 & 3,72 & 2,64 & 4,34 & 5,58 & - \\
\hline Average 2008- & 4,76 & 5,43 & 6,11 & 3,80 & 5,77 & 6,53 & - \\
\hline $\mathbf{2 0 1 3}$ & & & & & & & - \\
\hline Variation \% 2008- & 52,51 & 31,56 & 25,19 & 39,88 & $-19,48$ & 8,85 & - \\
\hline $\mathbf{2 0 1 3}$ & & & & & & & \\
\hline
\end{tabular}

Table 14: Selling Prices-Market of Germany. 


\section{Discussion}

Previous researches revealed the important place that Greece occupies in the trade of fishery products [12]. Especially exports of Greek aquaculture products seem to dominate in the EU-27 [12-17].

According to the results of this study, it is noted that the markets of the EU-27 are very demanding and constantly evolving in terms of competitiveness.

In particular, for the Italian market, it seems that the Greek sea bream holds the largest market share since 2000, followed by Turkey and Malta and after 2009 the new competitor country Croatia. In the period of 2000-2013 all the quantities of sea bream imported in Italy from Greece and the other competitor countries prove the dominant position of Greece, which from 8.700 tons in 2000 reached 19.500 tons in 2013. Turkey's sea bream follows with 2.500 tons and Malta's with 1.000 tons. Thus, the Italian market is the most important market for the Greek sea bream with value of imports in 2013, up to 81 million euros.

More specifically for the Italian market: Greece holds the first position in exports from 2000 up to 2013. Malta stands in second place with the highest competitive advantage. Croatia is a new competitor country after 2009. Turkish sea bream has the lowest price, of average 3.5 euros $/ \mathrm{kg}$, followed by Malta's and Greece's sea bream at 4 euros $/ \mathrm{kg}$. Regarding the rest importing markets, we conclude that over the last 6 years, from 2008 up to 2013:

Greece presents values greater than one, thus revealing a rather strong competitive advantage. Turkey holds the first place only in the Spanish market and second place in the markets of United Kingdom, Germany and Romania. As far as the Sale Prices of sea bream concerns, in average:

Greek sea bream is the first cheapest in France, Portugal and United Kingdom. Turkish sea bream is the firsts cheapest in Spain, Germany and Romania. The position of the Greek sea bream in all the studied import markets is:

First in six out of the seven markets in exports value, first in five out of the seven markets in competitive advantage, first lowest price in three out of the seven markets, second lowest price in the remaining four markets. Emerging in the market of Romania, Turkish sea bream is:

First lowest price in four out of the seven markets, second in four out of the seven markets in exports value, second in three out of the seven markets in competitive advantage, the main competitor of Greece.

Therefore, the Greek sea bream is characterized by a satisfactory degree of competitiveness in most of the studied importing markets. Regarding the analysis of competitor - exporting countries, Turkey constitutes a serious threat to Greek exports of sea bream. The lowest price usually accompanies the Turkish sea bream, which appears to be the most important factor for the continuous improvement of Turkey's competitive position against Greek exports.

The Greek exports should be directed to alternative marketing strategies, aiming at improving competitiveness, without focusing on the own price but in processes that will improve the added value, thus fulfilling better the continually changing preferences of the European consumers. This remark is compatible with previous studies, highlighting that the orientation of Greek exports to further development of remaking processes and improvement in marketing strategies, combined with the implementation of quality - assurance systems is the dynamic response to European and international challenges $[9,11]$.

In addition, it could be important for Greek sea bream exports to establish brand product strategies reinforcing the customer value. Considering that the collective fisheries sector is facing the acute problem of overfishing in recent years, the development of aquaculture is a very serious issue for our economy's development and the sustainable management of the aquatic environment. Therefore, adopting an integrated approach, according to the standards of the sustainable management systems, could be an efficient strategy for local production in facing the international challenges.

The shift to a healthy lifestyle and the increasing trend of fish consumption per capita, coupled with the stabilization or decline of collecting fisheries, highlights that conditions of aquaculture industry will be favorable in the future. Although the sector is facing serious threats, such as the possibility of strengthening of the competitive position of Turkey, or the emergence of new producer countries with lower labor costs close to major centers of consumption, the overall exogenous prospects are positive.

The need for better coordination between enterprises for joint interest actions is important. Better information about the market situation and the increased collaboration with research centers could significantly reduce the threat of falling prices, while improved and/or innovative products could be produced and promoted.

\section{Conclusions}

The main aim of this study was the investigation of the competitive position of Greek gilthead sea bream exports, in the market of EU-27.

The sea bream is one of the main export products of Greece, especially in the EU-27 market, reinforcing exports and improving its Balance of Trade.

Initially, the major importing countries-markets and the main competing countries, in terms of Exports were identified. Then, the competitive position and the evolution of the level of competitiveness of the Greek sea bream in each of the major importing markets were evaluated. The study performed using the index of Revealed Export Competitive Advantage (RXCA).

Results revealed that the main importing markets of Greek sea bream in the EU-27 are Italy, France, Portuguese, Spain, United Kingdom and Germany. In addition, an importing emerging market seems to be Romania, while the main competitive to Greek exporting countries seem to be Spain, Turkey, France, Italy, Malta and Croatia. Therefore, marketing strategies of Greek exports should be cautiously planned and continuously specialized in the particularities of the importing markets, aiming not only to improve individual characteristics of the products that are intended for exports to these countries, but also approaching and penetrating new emerging markets.

\section{References}

1. Karagounis C, Polymeros K (2012) Investigation of the competitiveness of Greek sea bream in the European Union market. Proceedings of the 11th PanHellenic Conference of Agricultural Economics, Thessaloniki.

2. Porter M (1998) The Competitive Advantage of Nations: with a new introduction London: Macmillan Press: 11-15, 33-39, 602. 
Citation: Oikonomou A, Polymeros K (2015) Analyzing the Competitiveness of the Greek Sea Bream Exports in the European Union Market. J Glob Econ 3: 145. doi:10.4172/2375-4389.1000145

3. Kim D Marion BW (1997) Domestic Market Structure and Performance in Global Markets: Theory and Empirical Evidence from US Food Manufacturing Industries. Review of Industrial Organization 12: 335-354.

4. Balassa B (1965) Trade liberalization and revealed comparative advantage Manchester School of Economic and Social Studies 1: $99-123$.

5. Lee $\mathrm{J}$ (1995) Comparative Advantage in Manufacturing as a Determinant of Industrialization: the Korean Case. World Development 23: 1195-1214.

6. Havrila I, Gunawardana P (2003) Analyzing Comparative Advantage and Competitiveness: an Application to Australia's Textile and Clothing Industries. Australian Economic Papers 42: 103-117.

7. Polymeros K, Loizou E, Tsakiridou R (2005) Estimating the Competitiveness Indices of the Greek Fisheries Products. Proceedings of the $8^{\text {th }}$ Pan-Hellenic Conference of Agricultural Economics, Agrotypos SA: 44-52.

8. Polymeros K, Tsakiridou E, Katrakylidis K (2005) Assessment of Market Shares of Greek exports of fishery products to the market of the EU. Proceedings of the 2nd Pan-Hellenic Conference of Hydrobiology - Fisheries (Challenges and Prospects in Marketing and Technology of seafood). Publishing Printing: 159-167.

9. Polymeros K, Katrakilidis K (2008) 'The Dynamic Characteristics of Competitiveness in the EU Fish Market'. The International Journal of Economic Issues 1: 25-42.

10. Polymeros K, Mattas K, Tsakiridou E (2005) Assessing the Competitiveness of EU Mediterranean Fisheries and Aquaculture Industries. 95th Seminar of the
European Association of Agricultural Economists (EAAE), The Economics of Aquaculture with Respect to Fisheries, Civitavecchia, Rome.

11. Polymeros KJ (2003) Manufacturing-Distribution and Marketing of fisheries products. Proceedings of the 1st Panhellenic Conference of Hydrobiology Fisheries (Fisheries-aquaculture conflicting or parallel activities).

12. Tsaklagkanos A (2004) Fundamentals of Marketing. Thessaloniki: Kyriakidis Brothers Publishing.

13. Kaimakoudi E, Polymeros K, Batzios Ch (2014) 'Investigating export performance of Balkan and Eastern European fisheries sector'. Procedia Economics and Finance 14: 219-230.

14. Karagounis C, Polymeros K (2011) Investigating the competitiveness of Greek sea bass in the EU market. Proceedings of the 4th International Symposium on Hydrobiology and Fisheries: 208-211.

15. Lioliou M (2009) Investigation of the competitiveness of Greek sea bream in the European Union market. Department of Agriculture Ichthyology and Aquatic Environment, University of Thessalyl.

16. Lioliou M, Polymeros K, Katrakylidis K (2010) The potential of Greek exports of animal products to the EU market. Proceedings of the 10th Panhellenic Conference of Agricultural Economics, Thessaloniki, Publisher Chart: 487-495.

17. Karagounis Ch (2011) The prospects of Greek fisheries products in the EU market. Unpublished MSc thesis, Department of Agriculture Ichthyology and Aquatic Environment, University of Thessaly, Volos. 